\title{
Nutzer- und Aufgabengerechte Unterstützung von Modellierungsaktivitäten im Kontext des MBSE-Model-Based Systems Engineering
}

Constantin Mandel, Matthias Behrendt, Albert Albers

MBSE-Model-Based Systems Engineering wird als vielversprechender Ansatz gesehen, die steigende Komplexität heutiger Produktentwicklungsprozesse beherrschbar zu machen. Bei der Einführung und Umsetzung von MBSE existieren jedoch hohe Einstiegshürden, insbesondere, wenn formale Modellierungssprachen wie SysML für Modellierungsaktivitäten verwendet werden. Gleichzeitig bieten formale Modellierungssprachen jedoch Vorteile bei der Ermöglichung einer domänenübergreifenden Kommunikation und dem Informationsaustausch. Dieser Beitrag beschreibt einen Ansatz zur nutzer- und aufgabengerechten Unterstützung von Modellierungsaktivitäten mittels formaler Modellierungssprachen im Kontext des MBSE. Ziel des Ansatzes ist es, die Modellierung auf die Bedarfe und Aufgaben einzelner Anwender bzw. deren Rolle im Produktentwicklungsprozess zu fokussieren und somit einen gezielteren Zugang zur Modellierung zu ermöglichen. Hierzu werden Ansätze aus dem Bereich Architekturframeworks zur Strukturierung von Modellen mittels Bedarfs-getriebener Sichten mit einer Aktivitäts-getriebenen Formalisierung von Methoden des MBSE kombiniert. Methoden des MBSE werden in einzelne Modellierungsaktivitäten unterteilt und diese mit Sichten aus einem Architekturframework, die die Modellierungsaktivität unterstützen, verknüpft. Somit wird einerseits eine klare Strukturierung des Modells nach Sichten und andererseits eine gezielte und eindeutige Durchführung bedarfsgerechter Methoden des MBSE ermöglicht.

Keywords: MBSE, Architekturframework, Modellierungsmethoden, Nutzer- und AufgabenZentrierung

\section{Einführung und Motivation}

Produktentwickler sehen sich mit beständig steigenden Kundenanforderungen, insbesondere hinsichtlich Funktionalität, Sicherheit und Umweltverträglichkeit, konfrontiert. Zudem verkürzen sich Produktlebenszyklen zunehmend, bei gleichzeitig steigendem Kostendruck. Dies führt zu erhöhter Komplexität in der Produktentwicklung und erfordert eine interdisziplinäre Zusammenarbeit verschiedener Domänen. (Beihoff et al. 2014) 
MBSE-Model-Based Systems Engineering wird als vielversprechender Ansatz gesehen, den beschriebenen Herausforderungen zu begegnen (vgl. bspw. Kleiner and Husung 2016). Systems Engineering im Allgemeinen und damit MBSE im Besonderen, findet jedoch noch keine flächendeckende Verbreitung in der Industrie (vgl. Stützel et al. 2018). Dies liegt unter anderem daran, dass das Erlernen und Anwenden von MBSEAnsätzen mit formalen, domänenübergreifenden Modellierungssprachen wie der SysML-Systems Modeling Language, oftmals eine hohe Einstiegshürde darstellen (vgl. z.B. Munker and Albers 2015; Alt 2019). Darüber hinaus muss die Einführung und Anwendung von MBSE-Methoden immer an die Situation und Bedarfe der Anwender angepasst werden, um individuelle und organisatorische Akzeptanz zu erreichen (vgl. Albers et al. 2012; Kleiner and Husung 2016; Delligatti 2014).

In diesem Beitrag wird ein Ansatz zur nutzer- und aufgabengerechten Unterstützung von Modellierungsaktivitäten im Kontext des MBSE vorgestellt. Die Motivation zu diesem Ansatz ergab sich aus Beobachtungen und Auswertungen bei der Einführung und Nutzung von MBSE im Live-Lab Integrierte Produktentwicklung (Albers et al. 2018). Der beschriebene Ansatz greift dabei bestehende Konzepte des MBSE wie Architekturframeworks sowie Modellierungsmethoden auf und integriert und erweitert diese.

\section{Stand der Forschung}

\section{(Model-Based) Systems Engineering}

MBSE beschreibt die formalisierte Erstellung konsistenter domänenübergreifender Systemmodelle zur Unterstützung von Anforderungsmanagement, Analyse, Verifikation und Validierung über den gesamten Produktlebenszyklus (International Council on Systems Engineering (INCOSE) - Technical Operations 2007).

Im Kontext des MBSE sind jeweils die drei Aspekte Modellierungssprache, Modellierungsmethoden und (Software-)Tool zur Modellierung integriert zu betrachten (Delligatti 2014). Als Modellierungssprache findet die SysML weite Verbreitung (Object Management Group 2017).

Im Zusammenspiel mit der gewählten Modellierungssprache beschreiben Modellierungsmethoden eine Abfolge von Schritten zur Erstellung der Systemmodelle (Delligatti 2014). Modellierungsmethoden sollten dabei stets gemäß des Ziels des Einsatzes von MBSE in einem Projekt gewählt werden (Delligatti 2014). Ein Anwendungsfall getriebenen Ansatz zur Fokussierung der Modellierung auf die vorliegende Problemstellung beschreiben bspw. Husung et al. (2018). 
In Literatur und Praxis lässt sich eine Vielzahl von Modellierungsmethoden zur Unterstützung des gesamten Produktentwicklungsprozesses (vgl. INCOSE Object-Oriented Systems Engineering Method (OOSEM) nach Friedenthal et al. (2012)) oder fokussiert für einzelne Aspekte innerhalb des Produktentwicklungsprozesses (bspw. die Functional Architecture for Systems Methode (FAS) zur Erarbeitung der funktionalen Architektur eines Systems ausgehend von Systemanforderungen (Lamm and Weilkiens 2010)) finden.

Eine Vielzahl existierender Methoden deuten zwar Iterationen und das „Springen“ zwischen einzelnen Teilschritten an, sind im Grunde jedoch eher sequentiell aufgebaut. Wie Albers beschreibt, sind Produktentwicklungsprozesse jedoch immer einzigartig und individuell (Albers 2010). Übergeordnete sequentielle Beschreibungen können somit zwar Rahmenwerke für die Entwicklung vorgeben, beschreiben aber nicht, was in unvorhergesehen oder individuellen Situationen in einem Produktentwicklungsprozess geschehen soll (Albers 2010).

Im integrierten Produktentstehungsmodell (iPeM) beschreibt Albers daher eine Reihe von wiederkehrenden Aktivitäten im Rahmen eines Produktentwicklungsprozesses. Im Gegensatz zu Phasen, sind die Aktivitäten unabhängig von spezifischen Zeitpunkten für Start und Ende. Im Kontext eines spezifischen Produktentstehungsprozesses können die Aktivitäten wiederkehrend und parallel in einem Phasenmodell angeordnet werden. (Albers 2010)

\section{Architekturframeworks}

Morkevicius et al. beschreiben, dass viele Methoden im Kontext des MBSE keine Rahmenwerke bzw. "Frameworks“ zur Organisation der Modelle und der Modellierung (bspw. in einem Software-Tool) vorgeben. Somit blieben die Modellierungsmethoden zu abstrakt zur Lösung konkreter Probleme. (Morkevicius et al. 2017)

Holt und Perry (Holt and Perry 2013) beschreiben einen Ansatz bestehend aus 3 Elementen zum Aufbau von Modellen im Kontext des MBSE:

- Eine Ontologie zur Definition der gemeinsamen Sprache bzw. Sprachelemente

- Sichten („Views“) als Ausschnitte eines Modells und Viewpoints als „Baupläne“ für die Sichten. Dies lehnt sich an die Beschreibungen der ISO 42010 Systems and software engineering - Architecture description an (42010).

- Ein Architekturframework zur Sammlung und Organisation der Sichten. Im weiteren Verlauf des Beitrags wird zur verallgemeinerten Beschreibung der Begriff Modellframework verwendet. 
In einem ähnlichen Verständnis werden in Literatur und Praxis inzwischen einige Modellframeworks beschrieben. Beispiele hierfür sind das MagicGrid nach Aleksandraviciene \& Morkevicius (2018) oder das SPES Framework nach Pohl et al. (2016).

\section{Forschungslücke und Forschungsfragen}

Wie in Absatz 0 beschrieben, werden viele Modellierungsmethoden im Kontext des MBSE eher sequentiell beschrieben und sind somit nicht ohne Weiteres praxisgerecht im Produktentwicklungsprozess anwendbar. Zusätzlich wird in vielen Methoden nicht beschrieben, wie diese in einer (Tool-) Implementierung umzusetzen sind, was die Anwendbarkeit entstehender Modelle beeinträchtigt. Zudem wird die Wiederverwendbarkeit entstehender Modelle eingeschränkt, da keine Angaben zu einer konsistenten Ablage von Informationen in verschiedenen Modellen gemacht werden, was das gezielte Auffinden und Nutzen spezifischer Informationen erschwert. Demgegenüber bieten Modellframeworks oftmals eher eine statische Sicht auf das Modell und machen alleine keine Aussage, wie Informationen zu dem Modell hinzugefügt, entfernt oder geändert werden sollen bzw. welche Sichten für verschiedene Anwendungsfälle zu nutzen sind.

Im Kontext dieses Beitrags sollen daher zwei zentrale Forschungsfragen untersucht werden:

- Wie kann eine Orientierung an Nutzern und Aufgaben für die Modellierung im Sinne des MBSE mit Hilfe eines Modellframeworks und Modellierungsmethoden realisiert werden?

- Wie lassen sich die (statische) Strukturierung des Modells über Sichten im Sinne eines Modellframeworks und die nutzer- und aufgabengerechte Auswahl und Durchführung von Modellierungsmethoden integrieren, um ein formalisiertes und wiederverwendbares Vorgehen bei der Nutzung von Systemmodellen zu ermöglichen?

\section{Ansatz}

Der in diesem Beitrag vorgestellte Ansatz kombiniert die Nutzung eines Modellframeworks mit der Definition problemspezifischer Modellierungsaktivitäten. Das beispielhafte Modellframework in Abbildung 1 ist in SysML implementiert und stellt einen Ordnungsrahmen für das Systemmodell zur Verfügung. In Anlehnung an das SPES-Frame- 
work (Pohl et al. 2016) ist das vorgestellte Modellframework zweidimensional über Systemebenen (Zeilen) und Perspektiven zur Ordnung der Viewpoints (Spalten) aufgebaut. Die weißen Rechtecke repräsentieren jeweils Diagramme zu den Viewpoints und verlinken auf entsprechende Darstellungen der Sichten. So wird beispielsweise im Diagramm zum Viewpoint Anwendungsfälle eine Beschreibung zu den dargestellten Informationen bzw. Hinweise zu deren Nutzung, Links zu passenden Sichten (in diesem Fall ein SysML-Anwendungsfalldiagramm) und Links zu Ressourcen bzw. Paketen aus der Modellstruktur (in diesem Fall der Ordner, welcher die Anwendungsfälle enthält) dargestellt (siehe Abbildung 2).

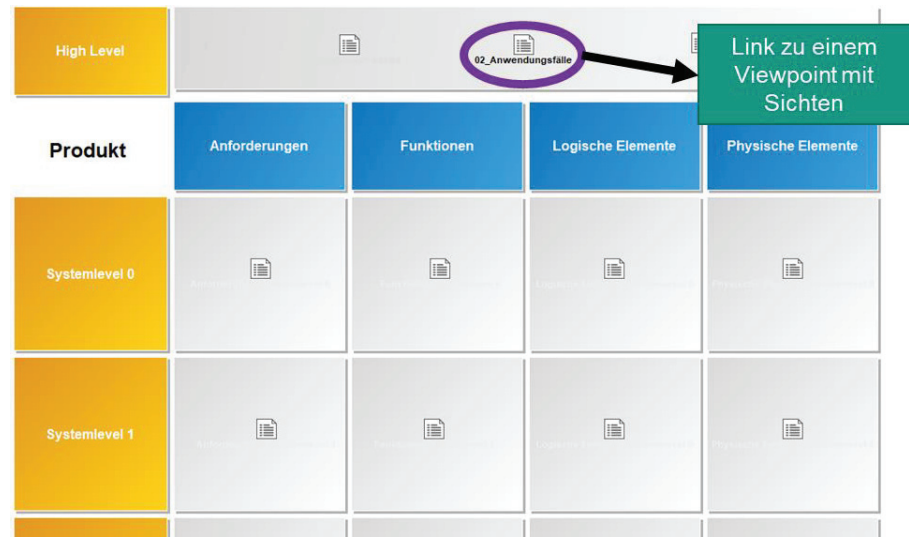

Abbildung 1: Beispielhaftes Modellframework (Ausschnitt)

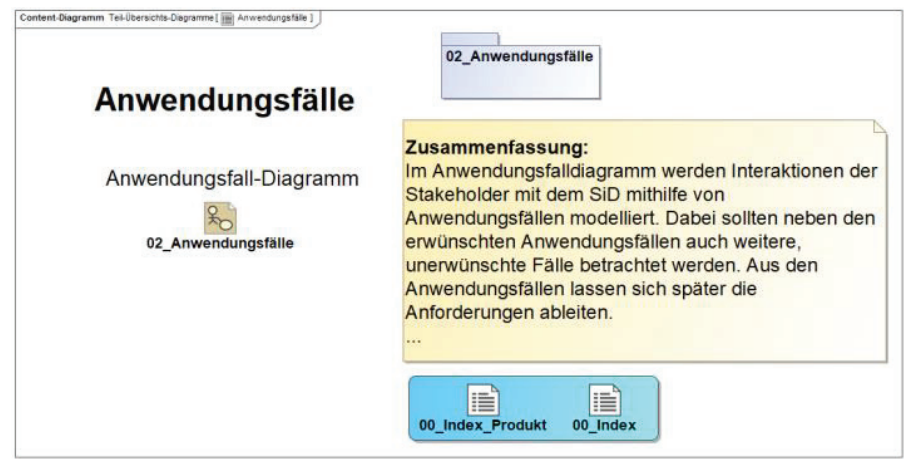

Abbildung 2: Darstellung des Diagramms zum Viewpoint "Anwendungsfälle" 
Die im vorgestellten Ansatz beschriebenen Modellierungsaktivitäten gliedern Modellierungsmethoden in wiederverwendbare Teil-Aktivitäten. Jede dieser Teil-Aktivitäten kann wiederum aus einem oder mehreren Schritten bestehen, die im Wechselspiel Analyse und Syntheseaktivitäten beschreiben. Die Schritte beschreiben dabei entweder Analyse bestehender Informationen aus dem Modell oder das Hinzufügen neuer Informationen zum Modell. Dabei ist jedem der Schritte eine Sicht aus dem Modellframework zugeordnet. Abbildung 3 zeigt beispielhaft den Ablauf der Modellierungsaktivität "Stakeholder Bedarfe herausarbeiten", welche in ähnlicher Form als Teil-Aktivität der OOSEM Methode nach Friedenthal et al. (2012) zu finden ist. Der Ablauf der Modellierungsaktivität ist im gleichen Modell wie das Modellframework als SysML-Aktivitätsdiagramm angelegt. Jeder Teilschritt der Modellierungsaktivität erhält einen Hyperlink zur entsprechenden Sicht aus dem Modellframework, bzw. einer Darstellung inklusive zusätzlicher Beschreibung zur Durchführung des Teilschrittes (Abbildung 3, rechts). Wird eine Sicht durch das Hinzufügen, Ändern oder Entfernen von Informationen angepasst, hat dies für gewöhnlich Auswirkung auf andere Teile des Modells. Durch die Nutzung des integrierten MBSE-Ansatzes werden diese Informationen in allen Sichten, die die entsprechende Informationen enthalten, angepasst. Die Schritte der Modellierungsaktivitäten stellen somit auch Links zu weiteren Sichten bereit, um die Auswirkung der gerade vorgenommenen Änderung am Modell auch in anderen Sichten, sprich Kontexten, zu überprüfen. Darauf aufbauend können weitere Maßnahmen zur Anpassung des Modells oder weitere Aktivitäten im Produktentwicklungsprozess angestoßen werden.

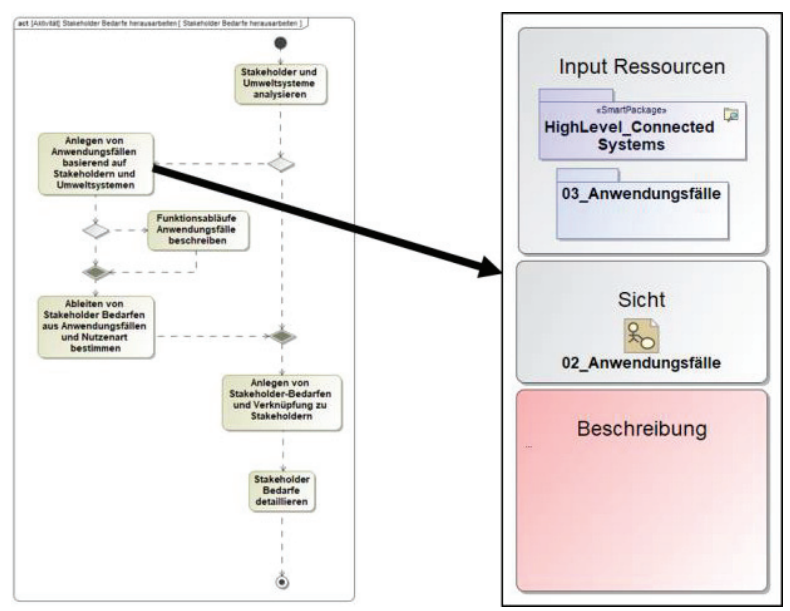

Abbildung 3: Ablauf der Modellierungsaktivität "Stakeholder Bedarfe herausarbeiten" 
Durch diese Kombination aus Modellframework und Modellierungsaktivitäten, kann eine nutzer- und aufgabengerechte Nutzung eines Systemmodells erreicht werden. Einerseits sind, orientiert am Verständnis der 42010 42010, die Sichten im Modellframework so angelegt, dass sie spezifisch die Bedarfe bestimmter Nutzer-/Rollengruppen adressieren. Dadurch kann je nach Bedarf eines Modellnutzers über das zentrale Modellframework gezielt zu einer spezifischen Sicht als Ausschnitt aus dem Gesamt-Modell navigiert werden. Die Aufgabenorientierung geschieht durch die Auswahl passender Modellierungsaktivitäten: Jedem Nutzer des Modells können in einem konkreten Produktentwicklungsprozess für dort anfallende Aufgaben die entsprechenden Modellierungsaktivitäten zugewiesen werden. Die Modellierungsaktivitäten werden dabei in einer Bibliothek zusammen mit dem Modellframework als wiederverwendbare Elemente angelegt.

Bei der Nutzung und Erstellung von Systemmodellen ist es dabei nicht immer notwendig, alle angelegten Modellierungsaktivitäten und Sichten zu nutzen. Je nach Aufgaben und Zielen der Modellnutzung sind bestimmte Aktivitäten und Sichten ggf. nicht relevant. Angelehnt an den Ansatz des Anwendungsfall-getriebenen MBSE nach Husung et al. (2018) können zum Anfang eines Produktentwicklungsprozesses die relevanten Modellierungsaktivitäten ausgewählt und das Modellframework somit nur mit den für die Modellierungsaktivitäten relevanten Sichten im Modell zur Verfügung gestellt werden. Im Kontext einer agilen Entwicklung kann die Auswahl relevanter Modellierungsaktivitäten pro Sprint stattfinden. Somit kann das Modell problemspezifisch sowie nutzer- und aufgabengerecht über den Produktentwicklungsprozess kontinuierlich befüllt werden und wachsen.

\section{Diskussion}

Die Entwicklung des vorgestellten MBSE-Ansatzes wurde durch Beobachtungen und Auswertungen bei der Einführung eines MBSE-Ansatzes im Live-Lab Integrierte Produktentwicklung (Albers et al. 2018) motiviert. Basierend auf einer Erweiterung des SysKIT-Schulungsansatzes (vgl. Matthiesen et al. 2014) wurden den Teilnehmern des Live-Labs sowohl ein Modellframework als auch eine Ablaufdiagramm der übergeordneten Modellierungsmethode in einer SysML-Modellvorlage zur Verfügung gestellt. Beide Darstellungen waren dabei mit Hilfe der gleichen Viewpoints aufgebaut. In einer Umfrage gaben die Teilnehmer überwiegend an, dass das bereitgestellte Modellframework beim Verständnis des Modellierungsansatzes unterstütze. Auf die Frage, ob die Matrix-förmige Darstellung des Frameworks (ähnlich der Darstellung in Abbildung 1) oder die sequentielle, Wasserfall-artige Darstellung der Sichten für die Modellierung 
genutzt wurde und zum Verständnis beigetragen habe, gab es eine klare Tendenz zur Wasserfall-artigen Darstellung. Eine derartige Darstellung suggeriert jedoch ein lineares Vorgehen in der Erstellung und Nutzung der Modelle und somit des Produktentwicklungsprozesses, was den in Absatz Obeschriebenen Erkenntnissen nach Albers (2010) entgegen steht.

Im Zuge des Projekts MecPro ${ }^{2}$ wurde ein ähnlicher Ansatz wie der in diesem Beitrag beschriebene entwickelt (vgl. Eigner et al. 2015). In MecPro ${ }^{2}$ wurde, basierend auf den Ansätzen nach Holt und Perry (vgl. Holt and Perry 2013), ein Modellframework und eine Reihe von Viewpoints entwickelt. Zusätzlich wurde die Verknüpfung zu einem Prozessrahmenwerk geschaffen. Dies geschieht durch die Zuweisung von Viewpoints zu Prozessrollen, die einzelne Personen im Produktentwicklungsprozess einnehmen. Die Viewpoints wiederum bestehen aus verschiedenen Sichten auf das Modell und werden den verschiedenen Prozessaktivitäten zugeordnet. (vgl. Eigner et al. 2015)

Der in diesem Beitrag vorgestellte Ansatz betrachtet hingegen in erster Linie nicht einen Ablauf von Prozessaktivitäten im Produktentwicklungsprozess, sondern Modellierungsmethoden im Sinne des MBSE zur Unterstützung der durch eine Projektplanung festgelegten Aktivitäten. Ziel ist es, die im Modell hinterlegten Modellierungsmethoden wiederkehrenden Aktivitäten im Produktentwicklungsprozess als Auswahl möglicher Unterstützungsmethoden zur Verfügung zu stellen. Die Zuordnung von Modellierungsmethoden zu den Aktivitäten des Produktentwicklungsprozesses geht damit über die Zuordnung eines Viewpoints zu einer Aktivität des Produktentwicklungsprozesses hinaus, da neben den spezifischen Sichten für die Aktivität auch ein methodisches Vorgehen zur Arbeit mit diesen zur Verfügung gestellt wird. Somit können hilfreiche Modellierungsmethoden bedarfsgerecht bei einer (agilen) Projektplanung für die anstehenden Aktivitäten des Produktentstehungsprozesses ausgewählt werden.

\section{Zusammenfassung und Ausblick}

In diesem Beitrag wird ein Ansatz zur Integration von Modellframeworks und Modellierungsmethoden im Sinne des MBSE vorgestellt. Ziel des Ansatzes ist es, eine nutzerund aufgabengerechte Modellierung zu unterstützen. Somit soll die Akzeptanz für die Anwendung von MBSE mit formalen Beschreibungssprachen gesteigert und die Einstiegshürden gesenkt werden. Gleichzeitig soll der Ansatz ein über Produktgeneration hinweg wiederverwendbares Vorgehen zur Erstellung und Nutzung von Systemmodellen unterstützen. Das im Beitrag vorgestellte Modellframework und die dargestellten Methoden sind dabei beispielhaft gewählt. Der vorgestellte Ansatz lässt sich auf verschiedene Modellframeworks und Methoden adaptieren und anwenden. 
Momentan laufen Forschungsarbeiten zur Nutzung des vorgestellten Ansatzes im Kontext eines agilen Projektmanagements. Ziel dabei ist es, die Auswahl relevanter Methoden in der Sprintplanung zu unterstützen und somit ein Systemmodell bedarfsgerecht aufzubauen, kontinuierlich zu erweitern und konsequent über den Produktentwicklungsprozess zu nutzen.

\section{Literaturverzeichnis}

Albers, Albert (2010): Five Hypotheses about Engineering Processes and their Consequences. In : Proceedings of the TMCE 2010.

Albers, Albert; Lohmeyer, Q:; Radimersky, A. (2012): Individuelle und organisatorische Akzeptanz von Methoden des Systems Engineering. Tag des Systems Engineering TdSE 2012, Paderborn. In Maik Maurer, Sven-Olaf Schulze (Eds.): Tag des Systems Engineering 2012. Tag des Systems Engineering. Paderborn. München: Carl Hanser.

Albers, Albert; Walter, B.; Wilmsen, M.; Bursac, N. (Eds.) (2018): Live-Labs as real-world validation environments for design methods. International Design Conference - Design 2018. DESIGN 2018.

Aleksandraviciene, Aiste; Morkevicius, Aurelijus (2018): MagicGrid Book of Knowledge. A Practical Guide to Systems Modeling using MagicGrid from No Magic. Edited by U. A.B. Vitae Litera. Kaunas, checked on 11/5/2020.

Alt, Oliver (2019): Modellbasiertes Systems Engineering ohne Anlernaufwand mit den Fundamental Modeling Concepts. In : Tag des Systems Engineering 2019, pp. 205-212, checked on 4/8/2020.

Beihoff, Bruce; Oster, Christopher; Friedenthal, Sanford; Paredis, Chris; Kemp, Duncan; Stoewer, Heinz et al. (2014): A World in Motion - Systems Engineering Vision 2025. Available online at https://www.incose.org/docs/defaultsource/aboutse/se-vision-2025.pdf, checked on 1/17/2019.

Delligatti, Lenny (2014): SysML distilled. A brief guide to the systems modeling language: Upper Saddle River, N]; Munich [u.a.] : Addison-Wesley.

Eigner, Martin; Dickopf, Thomas; Schulte, Tim; Schneider, Marc (Eds.) (2015): mecPro^2-Entwurf einer Beschreibungssystematik zur Entwicklung cybertronischer Systeme mit SysML. Tag des Systems Engineering. TdSE: Carl Hanser (2015).

Friedenthal, Sanford; Moore, Alan; Steiner, Rick; Lockheed Martin, The MathWorks, Raytheon (2012): A practical Guide to SysML: Morgan Kaufmann.

Holt, Jon; Perry, Simon (2013): SysML for Systems Engineering - 2nd Edition: A model-based approach: The Institution of Engineering and Technology.

Husung, Stephan; Lindemann, Gabriel; Korobov, Sergej; Hamester, Michael; Kleiner, Sven (2018): Use Case driven Modelbased Systems Engineering for industrial applications. In : Tag des Systems Engineering 2018, checked on 10/19/2020.

International Council on Systems Engineering (INCOSE) - Technical Operations (2007): INCOSE Systems Engineering Vision 2020. Edited by International Council on Systems Engineering (INCOSE). Available online at http://www.ccose.org/media/upload/SEVision2020_20071003_v2_03.pdf, checked on 1/17/2019.

42010: ISO/IEC/IEEE 42010 Systems and software engineering -- Architecture description, checked on 12/12/2019. 
Kleiner, Sven; Husung, Stephan (2016): Model Based Systems Engineering. Prinzipen, Anwendung, Beispiele, Erfahrung und Nutzen aus Praxissicht. In Sven-Olaf Schulze, Christian Tschirner, Rüdiger Kaffenberger (Eds.): Tag des Systems Engineering. Herzogenaurach, 25.-27. Oktober 2016. München: Carl Hanser Verlag GmbH \& Co. KG, pp. 13-22, checked on 1/17/2019.

Lamm, Jesko G.; Weilkiens, Tim (2010): Funktionale Architekturen in SysML. In GfSE-Gesellschaft für SystemsEngineering (Ed.): Proceedings des Tag des Systems Engineering (TdSE '10). München.

Matthiesen, Sven; Schmidt, Sebastian; Moeser, Georg; Munker, Florian (2014): The Karlsruhe SysKIT Approach - A Threestep SysML Teaching Approach for Mechatronic Students. In Procedia CIRP 21, pp. 385-390. DOl: 10.1016/j.procir.2014.03.136.

Morkevicius, Aurelijus; Aleksandraviciene, Aiste; Mazeika, Donatas; Bisikirskiene, Lina; Strolia, Zilvinas (2017): MBSE Grid: A Simplified SysML-Based Approach for Modeling Complex Systems. In INCOSE International Symposium 27 (1), pp. 136150. D0l: 10.1002/j.2334-5837.2017.00350.x.

Munker, F.; Albers, Albert (2015): SystemSketcher - Entstehung eines anwenderorientierten Ansatzes zur interdisziplinären Systemmodellierung. Tag des Systems Engineering. In S.-0. Schulze, C. Muggeo (Eds.): Tag des Systems Engineering 2015, Ulm 2015. Ulm: Carl Hanser.

Object Management Group (2017): OMG Systems Modeling Language SysML 1.5. Available online at https://www.omg.org/spec/SysML/About-SysML/, checked on 3/7/2019.

Pohl, Klaus; Broy, Manfred; Daembkes, Heinrich; Hönninger, Harald (2016): Advanced Model-Based Engineering of Embedded Systems. Cham: Springer International Publishing, checked on 12/9/2019.

Stützel, Benno; Borchardt, Laura; Illa, Thoams; Gerling, Christoph (2018): Studie "Systems Engineering in Deutschland". Die deutsche Unternehmenslandschaft im Vergleich. With assistance of Benno Stützel, Laura Borchardt, Thoams Illa, Christoph Gerling. Edited by Prozesswerk GmbH. Available online at http://www.prozesswerk.eu/site/assets/files/1179/se_studie_prozesswerk_doppelseiten.pdf, checked on 7/27/2020.

\section{Kontakt}

Constantin Mandel, M.Sc.

Dr.-Ing. Matthias Behrendt

Univ.-Prof. Dr.-Ing. Dr. h.c. Albert Albers

IPEK-Institut für Produktentwicklung

Karlsruher Institut für Technologie (KIT)

Kaiserstraße 10

76131 Karlsruhe

www.ipek.kit.edu 